\title{
LINEAR INDEPENDENCE OF THE RENORMALIZED HIGHER POWERS OF WHITE NOISE
}

\author{
L. ACCARDI \\ Centro Vito Volterra, Università di Roma Tor Vergata \\ via Columbia 2, 00133 Roma, Italy, E-mail: accardi@volterra.mat.uniroma2.it \\ http://volterra.mat.uniroma2.it
}

\begin{abstract}
A. BOUKAS
Department of Mathematics and Natural Sciences, American College of Greece Aghia Paraskevi, Athens 15342, Greece,E-mail: andreasboukas@acgmail.gr
\end{abstract}

The connection between the Lie algebra of the Renormalized Higher Powers of White Noise (RHPWN) and the centerless Virasoro (or Witt)-Zamolodchikov$w_{\infty}$ Lie algebras of conformal field theory, as well as the associated Fock space construction, have recently been established in Ref. ${ }^{1-6}$ In this note we prove the linear independence of the RHPWN Lie algebra generators.

\section{Introduction: Renormalized Higher Powers of White Noise}

The quantum white noise functionals $a_{t}^{\dagger}$ and $a_{t}$ satisfy the Boson commutation relations

$$
\left[a_{t}, a_{s}^{\dagger}\right]=\delta(t-s), ;\left[a_{t}^{\dagger}, a_{s}^{\dagger}\right]=\left[a_{t}, a_{s}\right]=0
$$

where $t, s \in \mathbb{R}$ and $\delta$ is the Dirac delta function, as well as the duality relation

$$
\left(a_{s}\right)^{*}=a_{s}^{\dagger}
$$

Here (and in what follows) $[x, y]:=x y-y x$ is the usual operator commutator. For all $t, s \in \mathbb{R}$ and integers $n, k, N, K \geq 0$ we have $\left(\right.$ Ref. $^{6}$ )

$$
\left[a_{t}^{\dagger^{n}} a_{t}^{k}, a_{s}^{\dagger^{N}} a_{s}^{K}\right]=
$$




$$
\begin{aligned}
& \epsilon_{k, 0} \epsilon_{N, 0} \sum_{L \geq 1}\left(\begin{array}{c}
k \\
L
\end{array}\right) N^{(L)} a_{t}^{\dagger^{n}} a_{s}^{\dagger}{ }^{N-L} a_{t}^{k-L} a_{s}^{K} \delta^{L}(t-s) \\
& -\epsilon_{K, 0} \epsilon_{n, 0} \sum_{L \geq 1}\left(\begin{array}{c}
K \\
L
\end{array}\right) n^{(L)} a_{s}^{\dagger^{N}} a_{t}^{\dagger n-L} a_{s}^{K-L} a_{t}^{k} \delta^{L}(t-s)
\end{aligned}
$$

where for $n, k \in\{0,1,2, \ldots\}$ we have used the notation $\epsilon_{n, k}:=1-\delta_{n, k}$, where $\delta_{n, k}$ is Kronecker's delta and $x^{(y)}=x(x-1) \cdots(x-y+1)$ with $x^{(0)}=1$. In order to consider the smeared fields defined by the higher powers of $a_{t}$ and $a_{t}^{\dagger}$, for a test function $f$ and $n, k \in\{0,1,2, \ldots\}$ we define the sesquilinear form

$$
B_{k}^{n}(f):=\int_{\mathbb{R}} f(t) a_{t}^{\dagger^{n}} a_{t}^{k} d t
$$

with involution

$$
\left(B_{k}^{n}(f)\right)^{*}=B_{n}^{k}(\bar{f})
$$

$\mathrm{In}^{1}$ and $^{2}$ we introduced the convolution type renormalization of the higher powers of the Dirac delta function

$$
\delta^{l}(t-s)=\delta(s) \delta(t-s) ; \quad l=2,3, \ldots
$$

By multiplying both sides of (3) by test functions $f(t) g(s)$ such that $f(0)=$ $g(0)=0$ and then formally integrating the resulting identity (i.e. taking $\iint \ldots d s d t$ of both sides), using (6), we obtained the RHPWN Lie algebra commutation relations

$$
\left[B_{k}^{n}(f), B_{K}^{N}(g)\right]_{R H P W N}:=(k N-K n) B_{k+K-1}^{n+N-1}(f g)
$$

As shown in ${ }^{1}$ and, ${ }^{2}$ for $n, k \in \mathbb{Z}$ with $n \geq 2$, the white noise operators

$$
\hat{B}_{k}^{n}(f):=\int_{\mathbb{R}} f(t) e^{\frac{k}{2}\left(a_{t}-a_{t}^{\dagger}\right)}\left(\frac{a_{t}+a_{t}^{\dagger}}{2}\right)^{n-1} e^{\frac{k}{2}\left(a_{t}-a_{t}^{\dagger}\right)} d t
$$

with involution 


$$
\left(\hat{B}_{k}^{n}(f)\right)^{*}=\hat{B}_{-k}^{n}(\bar{f})
$$

satisfy the commutation relations of the second quantized VirasoroZamolodchikov- $w_{\infty}$ Lie algebra (Ref. ${ }^{7}$ ), namely

$$
\left[\hat{B}_{k}^{n}(f), \hat{B}_{K}^{N}(g)\right]_{w_{\infty}}=(k(N-1)-K(n-1)) \hat{B}_{k+K}^{n+N-2}(f g)
$$

In particular,

$$
\hat{B}_{k}^{2}(f):=\int_{\mathbb{R}} f(t) e^{\frac{k}{2}\left(a_{t}-a_{t}^{\dagger}\right)}\left(\frac{a_{t}+a_{t}^{\dagger}}{2}\right) e^{\frac{k}{2}\left(a_{t}-a_{t}^{\dagger}\right)} d t
$$

is the white noise form of the centerless Virasoro algebra generators.

We may analytically continue the parameter $k$ in the definition of $\hat{B}_{k}^{n}(f)$ to an arbitrary complex number $k \in \mathbb{C}$ and to $n \geq 1$ and we can show (Ref. ${ }^{3}$ ) that the RHPWN and $w_{\infty}$ Lie algebras are connected through

$$
\hat{B}_{k}^{n}(f)=\frac{1}{2^{n-1}} \sum_{m=0}^{n-1}\left(\begin{array}{c}
n-1 \\
m
\end{array}\right) \sum_{p=0}^{\infty} \sum_{q=0}^{\infty}(-1)^{p} \frac{k^{p+q}}{p ! q !} B_{n-1-m+q}^{m+p}(f)
$$

and

$$
B_{k}^{n}(f)=\left.\sum_{\rho=0}^{k} \sum_{\sigma=0}^{n}\left(\begin{array}{c}
k \\
\rho
\end{array}\right)\left(\begin{array}{l}
n \\
\sigma
\end{array}\right) \frac{(-1)^{\rho}}{2^{\rho+\sigma}} \frac{\partial^{\rho+\sigma}}{\partial z^{\rho+\sigma}}\right|_{z=0} \hat{B}_{z}^{k+n+1-(\rho+\sigma)}(f)
$$

For $n \geq 1$ we define the $n$-th order RHPWN $*$-Lie algebras $\mathcal{L}_{n}$ as follows: (i) $\mathcal{L}_{1}$ is the $*$-Lie algebra generated by $B_{0}^{1}$ and $B_{1}^{0}$ i.e., $\mathcal{L}_{1}$ is the linear span of $\left\{B_{0}^{1}, B_{1}^{0}, B_{0}^{0}\right\}$ (ii) $\mathcal{L}_{2}$ is the $*$-Lie algebra generated by $B_{0}^{2}$ and $B_{2}^{0}$ i.e., $\mathcal{L}_{2}$ is the linear span of $\left\{B_{0}^{2}, B_{2}^{0}, B_{1}^{1}\right\}$ (iii) For $n \in\{3,4, \ldots\}, \mathcal{L}_{n}$ is the $*$-Lie algebra generated by $B_{0}^{n}$ and $B_{n}^{0}$ through repeated commutations and linear combinations. It consists of linear combinations of creation/annihilation operators of the form $B_{y}^{x}$ where $x-y=k n, k \in \mathbb{Z}-\{0\}$, and of number operators $B_{x}^{x}$ with $x \geq n-1$. Through white noise and norm compatibility considerations, the action of the RHPWN operators on $\Phi$ was defined in ${ }^{4}$ as 


$$
B_{k}^{n}(f) \Phi:= \begin{cases}0 & \text { if } n<k \text { or } n \cdot k<0 \\ B_{0}^{n-k}(f) \Phi & \text { if } n>k \geq 0 \\ \frac{1}{n+1} \int_{\mathbb{R}} f(t) d t \Phi & \text { if } n=k\end{cases}
$$

In what follows, for all integers $n, k$ we will use the notation $B_{k}^{n}:=B_{k}^{n}\left(\chi_{I}\right)$ where $I$ is some fixed subset of $\mathbb{R}$ of finite measure $\mu:=\mu(I)>0$. Moreover, for all $t \in[0,+\infty)$ and for all integers $n, k$ we will use the notation $B_{k}^{n}(t):=$ $B_{k}^{n}\left(\chi_{[0, t]}\right)$.

To avoid ghosts (i.e., vectors of negative norm) appearing in the cases $n \geq 3$ in the Fock kernels $\left\langle\left(B_{0}^{n}\right)^{k} \Phi,\left(B_{0}^{n}\right)^{k} \Phi\right\rangle$ where $k \geq 0$, in ${ }^{4}$ we defined

$$
B_{n-1}^{n-1}\left(B_{0}^{n}\right)^{k} \Phi:=\left(\frac{\mu}{n}+k n(n-1)\right)\left(B_{0}^{n}\right)^{k} \Phi
$$

and were able to show that for all $k, n \geq 1$

$$
\left\langle\left(B_{0}^{n}\right)^{k} \Phi,\left(B_{0}^{n}\right)^{m} \Phi\right\rangle=\delta_{m, k} k ! n^{k} \prod_{i=0}^{k-1}\left(\mu+\frac{n^{2}(n-1)}{2} i\right)
$$

Therefore, the $\mathcal{F}_{n}$ inner product $\left\langle\psi_{n}(f), \psi_{n}(g)\right\rangle_{n}$ of the exponential vectors

$$
\psi_{n}(\phi):=\prod_{i} e^{a_{i} B_{0}^{n}\left(\chi_{I_{i}}\right)} \Phi
$$

where $\phi:=\sum_{i} a_{i} \chi_{I_{i}}$ is a test function, for $n=1$ is

$$
\left\langle\psi_{1}(f), \psi_{1}(g)\right\rangle_{1}:=e^{\int_{\mathbb{R}} \bar{f}(t) g(t) d t}
$$

while for $n \geq 2$ it is

$$
\left\langle\psi_{n}(f), \psi_{n}(g)\right\rangle_{n}:=e^{-\frac{2}{n^{2}(n-1)} \int_{\mathbb{R}} \ln \left(1-\frac{n^{3}(n-1)}{2} \bar{f}(t) g(t)\right) d t}
$$

where $|f(t)|<\frac{1}{n} \sqrt{\frac{2}{n(n-1)}}$ and $|g(t)|<\frac{1}{n} \sqrt{\frac{2}{n(n-1)}}$.

The $n$-th order truncated RHPWN (or TRHPWN) Fock space $\mathcal{F}_{n}$ is the Hilbert space completion of the linear span of the exponential vectors $\psi_{n}(f)$ 
under the inner product $\langle\cdot, \cdot\rangle_{n}$. The full TRHPWN Fock space $\mathcal{F}$ is the direct sum of the $\mathcal{F}_{n}$ 's.

The Fock representation of the TRHPWN generators $B_{0}^{n}$ and $B_{n}^{0}$ obtained in $^{4}$ is

$$
\begin{aligned}
& B_{n}^{0}(f) \psi_{n}(g)=n \int_{\mathbb{R}} f(t) g(t) d t \psi_{n}(g)+\left.\frac{n^{3}(n-1)}{2} \frac{\partial}{\partial \epsilon}\right|_{\epsilon=0} \psi_{n}\left(g+\epsilon f g^{2}\right) \\
& B_{0}^{n}(f) \psi_{n}(g)=\left.\frac{\partial}{\partial \epsilon}\right|_{\epsilon=0} \psi_{n}(g+\epsilon f)
\end{aligned}
$$

where $f:=\sum_{i} a_{i} \chi_{I_{i}}$ and $g:=\sum_{i} b_{i} \chi_{I_{i}}$ with $I_{i} \cap I_{j}=\oslash$ for $i \neq j$ and $f(0)=g(0)=0$.

As shown in, ${ }^{4}$ for all $s \in[0, \infty)$

$$
\left\langle e^{s\left(B_{0}^{1}(t)+B_{1}^{0}(t)\right)} \Phi, \Phi\right\rangle_{1}=e^{\frac{s^{2}}{2} t}
$$

i.e., $\left\{x_{1}(t):=B_{0}^{1}(t)+B_{1}^{0}(t)\right\}_{t \geq 0}$ is Brownian motion, while for $n \geq 2$

$$
\left\langle e^{s\left(B_{0}^{n}(t)+B_{n}^{0}(t)\right)} \Phi, \Phi\right\rangle_{n}=\left(\sec \left(\sqrt{\frac{n^{3}(n-1)}{2}} s\right)\right)^{\frac{2 n t}{n^{3}(n-1)}}
$$

i.e., for each $n \geq 2,\left\{x_{n}(t):=B_{0}^{n}(t)+B_{n}^{0}(t)\right\}_{t \geq 0}$ is a continuous binomial/Beta process.

\section{Linear independence of the RHPWN generators}

\section{Lemma 2.1.}

For all integers $m \geq 0$

$$
\sum_{n=0}^{m} c_{n} B_{0}^{n}\left(f_{n}\right)=0 \Longrightarrow c_{n}=0 \quad \forall n \in\{0,1, \ldots, m\}
$$

where we assume that the test functions $f_{n}$ are such that for all $n \in$ $\{0,1, \ldots, m\}$

$$
\int_{\mathbb{R}} f_{n}(t) a_{t}^{\dagger^{n}} d t \not \equiv 0
$$




\section{Proof.}

For $m=0$,

$$
c_{0} B_{0}^{0}\left(f_{0}\right)=0 \Longrightarrow c_{0} \int_{\mathbb{R}} f_{0}(t) d t=0 \Longrightarrow c_{0}=0
$$

and so (24) holds. Suppose that it holds for $m=M$. We will show that it is true for $m=M+1$ also. So suppose that

$$
\sum_{n=0}^{M+1} c_{n} B_{0}^{n}\left(f_{n}\right)=0
$$

Then

$$
\sum_{n=0}^{M+1} c_{n}\left[B_{1}^{0}(g), B_{0}^{n}\left(f_{n}\right)\right]=0
$$

where $g$ is any test function such that

$$
\int_{\mathbb{R}} g(t) f_{n}(t) a_{t}^{\dagger^{n}} d t \not \equiv 0
$$

for all $n$, i.e.,

$$
\sum_{n=0}^{M+1} n c_{n} B_{0}^{n-1}\left(g f_{n}\right)=0
$$

which is equivalent to

$$
\sum_{n=1}^{M+1} n c_{n} B_{0}^{n-1}\left(g f_{n}\right)=0
$$

or, letting $N:=n-1$, to

$$
\sum_{N=0}^{M}(N+1) c_{N+1} B_{0}^{N}\left(g f_{N+1}\right)=0
$$

which, by the induction hypothesis, implies that

$$
(N+1) c_{N+1}=0 \Longrightarrow c_{N+1}=0 \Longrightarrow c_{n}=0
$$


for all $n \in\{1,2, \ldots, M+1\}$. But then (27) reduces to $c_{0} B_{0}^{0}\left(f_{0}\right)=0$ which, as we have already seen, implies that $c_{0}=0$ as well.

Lemma 2.2. For all integers $m \geq 0$

$$
\sum_{k=0}^{m} c_{k} B_{k}^{0}\left(f_{k}\right)=0 \Longrightarrow c_{k}=0 \quad \forall k \in\{0,1, \ldots, m\}
$$

where we assume that the arbitrary test functions $f_{k}$ are such that for all $k \in\{0,1, \ldots, m\}$

$$
\int_{\mathbb{R}} f_{k}(t) a_{t}^{k} d t \not \equiv 0
$$

Proof. Taking the adjoint of equation (34) we obtain

$$
\sum_{k=0}^{m} \bar{c}_{k} B_{0}^{k}\left(\bar{f}_{k}\right)=0
$$

which by Lemma 2.1 implies that $\bar{c}_{k}=0$, and so $c_{k}=0$, for all $k \in$ $\{0,1, \ldots, m\}$.

Theorem 2.1. The generators $B_{k}^{n}(f)$ of the RHPWN Lie algebra are linearly independent, i.e., for all integers $m \geq 0$

$$
\sum_{n=0}^{m} \sum_{k=0}^{m} c_{n, k} B_{k}^{n}\left(f_{n, k}\right)=0 \Longrightarrow c_{n, k}=0 \quad \forall n, k \in\{0,1, \ldots, m\}
$$

where we assume that the arbitrary test functions $f_{n, k}$ are such that

$$
\int_{\mathbb{R}} f_{n, k}(t) a_{t}^{\dagger^{n}} a_{t}^{k} d t \not \equiv 0
$$

Note: By filling in with zero coefficients if necessary, every finite linear combination of the RHPWN generators can be put in the form

$$
\sum_{n=0}^{m} \sum_{k=0}^{m} c_{n, k} B_{k}^{n}\left(f_{n, k}\right)
$$


Proof. We will proceed by induction on $m$. For $m=0$, equation (37) becomes

$$
c_{0,0} B_{0}^{0}\left(f_{0,0}\right)=0 \Longrightarrow c_{0,0}=0
$$

which is true by (38). Suppose that equation (37) holds for $m=M$. We will show that it is true for $m=M+1$ also. So suppose that

$$
\sum_{n=0}^{M+1} \sum_{k=0}^{M+1} c_{n, k} B_{k}^{n}\left(f_{n, k}\right)=0
$$

Taking the commutator of (41) first with $B_{0}^{1}(g)$ and then with $B_{1}^{0}(g)$, where $g$ is any test function such that

$$
\int_{\mathbb{R}} g(t) f_{n, k}(t) a_{t}^{\dagger^{n}} a_{t}^{k} d t \not \equiv 0
$$

for all $n, k$, we obtain

$$
\sum_{n=0}^{M+1} \sum_{k=0}^{M+1} k n c_{n, k} B_{k-1}^{n-1}\left(g^{2} f_{n, k}\right)=0
$$

which is equivalent to

$$
\sum_{n=1}^{M+1} \sum_{k=1}^{M+1} k n c_{n, k} B_{k-1}^{n-1}\left(g^{2} f_{n, k}\right)=0
$$

which, letting $N:=n-1$ and $K:=k-1$, is equivalent to

$$
\sum_{N=0}^{M} \sum_{K=0}^{m}(K+1)(N+1) c_{N+1, K+1} B_{K}^{N}\left(g^{2} f_{N+1, K+1}\right)=0
$$

which, by the induction hypothesis, implies that

$$
(K+1)(N+1) c_{N+1, K+1}=0 \Longrightarrow c_{N+1, K+1}=0 \Longrightarrow c_{n, k}=0
$$

for all $n, k \in\{1,2, \ldots, M+1\}$. If $n=0$ and/or $k=0$ then equation (41) reduces to 


$$
c_{0,0} B_{0}^{0}\left(f_{0,0}\right)+\sum_{n=1}^{m+1} c_{n, 0} B_{0}^{n}\left(f_{n, 0}\right)+\sum_{k=1}^{m+1} c_{0, k} B_{k}^{0}\left(f_{0, k}\right)=0
$$

Taking the commutator of (47) with $B_{0}^{1}(g)$, where $g$ is as above, we obtain

$$
\sum_{k=1}^{m+1} k c_{0, k} B_{k-1}^{0}\left(g f_{0, k}\right)=0
$$

which by Lemma 2.2 implies that $k c_{0, k}=0$ for all $k \in\{1,2, \ldots, M+1\}$ and so $c_{0, k}=0$ for all $k \in\{1,2, \ldots, M+1\}$. Similarly, taking the commutator of (47) with $B_{1}^{0}(g)$ we obtain

$$
\sum_{n=1}^{m+1} n c_{n, 0} B_{0}^{n-1}\left(g f_{n, 0}\right)=0
$$

which by Lemma 2.1 implies that $n c_{n, 0}=0$ for all $n \in\{1,2, \ldots, M+1\}$ and so $c_{n, 0}=0$ for all $n \in\{1,2, \ldots, M+1\}$. So, (41) reduces to

$$
c_{0,0} B_{0}^{0}\left(f_{0,0}\right)=0
$$

which by (38) implies that $c_{0,0}=0$. Therefore $c_{n, k}=0$ for all $n, k \in$ $\{0,1,2, \ldots, M+1\}$.

\section{References}

1. L. Accardi and A. Boukas, Renormalized higher powers of white noise (RH$P W N)$ and conformal field theory, Infinite Dimensional Anal. Quantum Probab. Related Topics 9 (3) (2006), 353-360.

2. L. Accardi and A. Boukas, The emergence of the Virasoro and $w_{\infty}$ Lie algebras through the renormalized higher powers of quantum white noise, International Journal of Mathematics and Computer Science 1 (3) (2006), 315-342.

3. L. Accardi and A. Boukas, Renormalized Higher Powers of White Noise and the Virasoro-Zamolodchikov- $w_{\infty}$ Algebra, Reports on Mathematical Physics, 61 (1) (2008), 1-11, http://arxiv.org/hep-th/0610302.

4. L. Accardi and A. Boukas, Fock representation of the renormalized higher powers of white noise and the Virasoro-Zamolodchikov- $w_{\infty} *$-Lie algebra, to appear in Journal of Physics A: Mathematical and Theoretical, arXiv:0706.3397v2 [math-ph].

5. L. Accardi and A. Boukas, Lie algebras associated with the renormalized higher powers of white noise, Communications on Stochastic Analysis 1 (1) (2007), 57-69. 
10 L. Accardi \& A. Boukas

6. L. Accardi, A. Boukas and U. Franz, Renormalized powers of quantum white noise, Infinite Dimensional Analysis, Quantum Probability, and Related Topics, 9 (1) (2006), 129-147.

7. S. V. Ketov, Conformal field theory, World Scientific, 1995. 